\title{
Coupled Mode Theory of Electron-Beam Parametric Amplification
}

\author{
R. W. GouLD \\ California Instilule of Technology, Pasadena, California \\ AND \\ C. C. Johnson \\ Hughes Research Laboratories, Malibu, California
}

(Received August 19, 1960)

\begin{abstract}
A theory of parametric amplification in a filamentary electron beam by transverse fields is developed in coupled mode form. Space charge effects are neglected. In addition to beam modes at the signal frequency, beam modes at frequencies $\omega_{n}=\omega+\omega_{p}, n=0, \pm 1, \pm 2 \cdots$, where $\omega_{p}$ is the pump frequency, are coupled together. A discussion of the general form of the equations is given and reveals the circumstances under which exponential gain or periodic energy transfer between various modes can occur. When applied to quadrupole electric pump fields, a description of the quadrupole amplifiers of Adler, Wade, and Gordon is obtained. This theory is then used to evaluate the noise contribution from synchronous beam modes and higher cyclotron idler modes. Coupling by axially symmetric electric fields and by axially symmetric magnetic fields is discussed, and other amplification schemes suggested. The theory of coupling by axially symmetric fields can also be used to study lens effects on noise in the gun region.
\end{abstract}

\section{INTRODUCTION}

$\mathbf{I}^{\mathrm{N}}$ $\mathrm{N}$ the past few years there has been considerable interest and activity in parametric amplifiers for low noise amplification. One of the most successful of these is the Adler-Wade quadrupole amplifier, ${ }^{1}$ in which an electron beam is subjected to a time-varying but spatially uniform quadrupole electric field. Gordon has suggested several variations, including pumping by a spatially varying dc quadrupole electric field. ${ }^{2}$ The purpose of this paper is to present a general theory of these devices together with several new results. In addition, the theory is generalized to include the effect of axially symmetric transverse fields such as are encountered in the gun region. From the form of the equations, new amplification and energy transfer schemes are apparent.

The description of microwave tube operation in terms of coupling of modes of propagation has proved to be of great conceptual value $e^{3,4}$ and also has certain computational advantages. ${ }^{5,6}$ This paper will show that this approach can be extended to include transverse-field beam-type parametric amplifiers. Longitudinal-field beam-type parametric amplifiers ${ }^{7}$ can be treated in the same manner. ${ }^{8,9}$ Since certain coupled mode concepts and mathematical relations will be used repeatedly, they are summarized in this section.

\footnotetext{
${ }^{1}$ R. Adler, G. Hrbek, and G. Wade, Proc. I.R.E. 47, 1713 (1959).

2 E. I. Gordon, Proc. I.R.E. 48, 1158 (1960).

3 J. R. Pierce, Bell System Tech. J. 33, 1343 (1954).

4 R. W. Gould, I.R.E. Trans, on Electron Devices ED-2, 37 (1955).

${ }^{5}$ R. W. Gould, Proc. I.R.E. 47, 419 (1959).

${ }^{6}$ A. Ashkin, W. H. Louisell, and J. S. Cook, J. Electronics and Control 7, 1 (1960).

${ }^{7}$ W. H. Louisell and C. F. Quate, Proc. I.R.E. 46, 707 (1958).

${ }^{8}$ J. S. Cook and W. H. Louisell, Proc. I.R.E. 47, 2016 (1959).

${ }^{9}$ D. C. Forster, Ph.D. thesis, California Institute of Technology, Pasadena, California, June, 1960.
}

In previous analyses of parametric amplifiers, ${ }^{7,10}$ one finds that the complex conjugates of certain variables arise out of the condition that all physical quantities be real. Although the conjugate variables present no essential difficulty, we prefer to avoid them. Since the equations which are to be solved are linear with real variable coefficients, one can assume exponential time dependence $\left(\sum_{n} a_{n} e^{j \omega_{n} t}\right)$ and simply take the real part of the complex expression to obtain the physical result. In doing so, positive and negative frequencies arise. For example, in the Adler-Wade fast cyclotron wave amplifier, if the signal frequency is $\omega$, the idler frequency is $\omega-\omega_{p} \approx-\omega$, where $\omega_{p} \approx 2 \omega$ is the pump frequency.

The differential equations describing disturbances in traveling wave tubes can be writtin in terms of mode amplitudes ${ }^{5,6,11}$

$$
j\left(\partial a_{r} / \partial z\right)-\sum_{s} H_{r s} a_{s}=0
$$

where $a_{r}$ is the amplitude of the $r$ th mode, $H_{r r}$ is the propagation constant of the $r$ th mode without coupling, and terms for which $r \neq s$ are responsible for coupling between modes. In matrix notation,

$$
j(\partial a / \partial z)-H a=0
$$

where $a(z)$ is a vector with components $a_{r}(z)$, and $H$ is a square matrix with elements $H_{r g}$. We seek normal mode solutions of Eq. (2), in which each component has the same $z$ variation:

$$
a(z)=A e^{-j \beta z},
$$

where $A$ is a constant vector with components $A_{r}$. With this assumption, Eq. (2) takes the form of an eigenvalue problem:

$$
H A=\beta A,
$$

${ }^{10} \mathrm{H}$. Heffner and G. Wade, J. Appl. Phys. 29, 1321 (1958).

${ }^{11}$ A. E. Siegman, J. Appl. Phys. 31, 17 (1960). 
in which the eigenvalues $\beta$ are propagation constants of the coupled modes. In the works cited in footnotes 5,6 , and 11 , and in this paper, it is found that $H$ is a real matrix with a symmetry property ${ }^{12}$

$$
H_{i j}= \pm H_{j i}
$$

the upper $(+)$ sign applying if the kinetic powers of modes $i$ and $j$ are both positive or both negative, and the lower (-) sign applying if the kinetic powers of modes $i$ and $j$ are different. By using the parity matrix $P$ of Haus and Robinson, ${ }^{13}$ this result is written

$$
P H=\widetilde{H} P \quad \text { or } \quad H P=P \tilde{H},
$$

where $\sim$ denotes the transpose. $P$ is a diagonal matrix with elements $P_{i i}=+1$ if mode $i$ carries positive power and $P_{i i}=-1$ if mode $i$ carries negative power. We will show that the equations of a beam-type parametric amplifier can be cast in precisely the same form, with suitably defined mode amplitudes for the idler frequencis as well as for the signal frequency.

Equation (2) is formally identical with the Schrödinger equation

$$
H \psi=(j / h)(\partial \psi / \partial t)
$$

and in solving Eq. (2) some of the same methods as are used in solving Eq. (7) will be employed. There is one significant difference, however. The operator $H$ in Eq. (7) is Hermitian, and hence its eigenvalues (the energy levels of the system) are real. The operator $H$ in Eq. (2) is not Hermitian, however, and in general the eigenvalues are not real. Complex eigenvalues describe growing or decaying waves.

From Eqs. (2) and (6) it is readily shown that

$$
(\partial / \partial z)\left(a^{*} P a\right)=0 .
$$

If the mode amplitudes are suitably defined, one can interpret Eq. (8) as saying that the power flow $a^{*} P a$ is independent of $z$ (Chu's power theorem). When parametric amplification is discussed (Sec. III) it is found convenient to define the mode amplitudes in such a way that their square gives kinetic power divided by frequency. Equation (8) can then be regarded as an extension of the Manley-Rowe power relations ${ }^{14}$ to electron beam devices.

Let us assume that Eq. (4) has been solved for the eigenvalues $\beta_{i}$ and the eigenvectors $A^{i}$. The eigenvalue $\beta_{i}$ is just the propagation constant of the $i$ th mode of the coupled system, and the components of the eigenvector $A^{i}$ give the relative amplitudes of uncoupled modes present in mode $i$ of the coupled system. A

\footnotetext{
${ }^{12}$ In problems where $H$ is complex, the symmetry property is $H_{i j}= \pm H_{i j}{ }^{*}$. The relations corresponding to $\mathrm{Eq}$. (6) become $P H=H^{+} P$ and $H P=P H^{+}$.

${ }^{13}$ H. A. Haus and F. N. H. Robinson, Proc. I.R.E. 43, 981 (1955).

${ }^{14}$ J. M. Manley and H. E. Rowe, Proc. I.R.E. 44, 904 (1956).
}

general solution of Eq. (2) may then be written

$$
a(z)=\sum_{i} C_{i} \Lambda^{i} e^{-j \beta_{i} z}
$$

where $C_{i}$ are constants determined by the initial conditions $a(0)$. The constants $C_{i}$ are easily determined by making use of the orthogonality of the eigenvectors ${ }^{15}$ :

We find that

$$
\left(\beta_{j}-\beta_{i}\right) A^{j} P A^{i}=0 .
$$

$$
\begin{aligned}
C_{i} & =A^{i} P a(0) / A^{i} P A^{i} \\
a(z) & =\sum_{i} \frac{A^{i} P a(0)}{A^{i} P A^{i}} e^{-j \beta_{i} z} .
\end{aligned}
$$

Following the works cited in footnotes 5 and 11, we let $M$ be the matrix relating $a(L)$ to $a(0)$, i.e.,

$$
a(L)=M a(0) .
$$

Comparing with Eq. (12), we obtain explicit expressions for the matrix elements:

$$
M_{r s}=\sum_{i} \frac{A_{r}^{i} P_{s s} A_{s}{ }^{i}}{A^{i} P A^{i}} e^{-j \beta_{i} L},
$$

where $A_{r}{ }^{i}$ is the $r$ th component of the $i$ th eigenvector $A_{i}$. This method of evaluation of $C_{i}$ has a considerable advantage over the usual methods (simultaneous equations) if the number of modes is large.

\section{Solution of the Coupled Mode Equations}

When only two modes are coupled together, the solution of the preceding equations is quite simple, and the results have already been given ${ }^{3,4}$ Several of the important results are simply summarized in terms of the notation employed here. The two eigenvalues of Eq. (4) are

$$
\begin{aligned}
& \beta_{1}=\frac{H_{11}+H_{22}}{2}+\left[\left(\frac{H_{11}-H_{22}}{2}\right)^{2}+H_{12} H_{21}\right]^{\frac{1}{2}} \\
& \beta_{2}=\frac{H_{11}+H_{22}}{2}-\left[\left(\frac{H_{11}-H_{22}}{2}\right)^{2}+H_{12} H_{21}\right]^{\frac{1}{2}} .
\end{aligned}
$$

When the parity of the modes is the same, the square root is always real and both propagation constants are real. The two modes describe a periodic interchange of energy between modes. Coupling which results in periodic interchange in energy is termed passive coupling. ${ }^{16}$ When the two modes have opposite parity $H_{12} H_{21}$ is negative, and if $\left[\left(H_{11}-H_{22}\right) / 2\right]^{2}<\left|H_{12} H_{21}\right|$, one of the resulting waves grows and the other decays with distance. When coupling between two modes can result in growing waves, the coupling is termed active. ${ }^{16}$

\footnotetext{
${ }_{15}$ This relation can be proved by using Eqs. (4) and (6) in the usual manner.

${ }_{10}$ W. H. Louisell and J. S. Cook, Wescon Convention Record, Los Angeles, California (1959).
} 
The situation to which this method will be applied is one in which two modes of opposite parity (for example, $P_{11}=+1, P_{22}=-1$ ) have the same unperturbed propagation constant $\left(H_{11}=H_{22}\right)$ in the presence of a number of other modes which have rather different unperturbed propagation constants. For simplicity we consider a single additional mode $\left(H_{33}\right)$ and assume that $\left|H_{13} H_{31}\right|,\left|H_{23} H_{32}\right|$, and $\left|H_{12} H_{21}\right|$ are much less than $\left(H_{33}-H_{11}\right)^{2} / 4$. Thus modes 1 and 2 are in synchronism and strongly coupled to each other, but only weakly coupled to mode 3 . We can therefore treat mode 3 as a perturbation. The effect of additional weakly coupled modes is obtained by superposition.

Primary interest centers on the matrix element $M_{13}$. In general, noise will not be stripped from mode 3 and a small fraction of this noise may be transferred to mode 1 (which is taken to be the fast cyclotron signal mode when this result is applied to the quadrupole amplifier). According to Eq. (14),

$$
M_{13} \simeq A_{1}{ }_{1} P_{33} A_{3}{ }^{1} e^{-j \beta_{1} L} \quad\left(P_{33}= \pm 1\right),
$$

where we have retained only the contribution from the growing wave $\beta_{1}$, since it is by far the largest, and $A^{1}$ is the normalized eigenvector (i.e., $A^{i} P A^{i}=1$ ) for the growing wave. The corresponding matrix element which gives the gain in the region under consideration is

The ratio

$$
M_{11} \cong A_{1}{ }^{1} P_{11} A_{1}{ }^{1} e^{-j \beta_{1} L} \quad\left(P_{11}=+1\right) .
$$

$$
M_{13} / M_{11}=P_{33}\left(A_{3}{ }^{1} / A_{1}{ }^{1}\right)
$$

gives directly a measure of the effectiveness of mode 3 in setting up the growing wave relative to that of mode 1. To evaluate this ratio approximately, use is made of Eq. (4) in component form:

$$
\begin{aligned}
& \left(H_{11}-\beta\right) A_{1}+H_{12} A_{2}+H_{13} A_{3}=0 \\
& H_{21} A_{1}+\left(H_{22}-\beta\right) A_{2}+H_{23} A_{3}=0 \\
& H_{31} A_{1}+H_{32} A_{2}+\left(H_{33}-\beta\right) A_{3}=0 .
\end{aligned}
$$

To find the propagation constant corresponding to the growing wave the third mode is first neglected completely $\left(H_{13}=H_{23}=0\right)$; one obtains, for the case $H_{11}=H_{22}$,

$$
\begin{aligned}
\beta_{1} & =H_{11}+j\left|H_{12}\right| \\
A_{2}{ }^{1} & =-j\left(\left|H_{12}\right| / H_{12}\right) A_{1}{ }^{1} .
\end{aligned}
$$

Substitution of Eqs. (20a) and (20b) into (19c) gives an approximate expression for the ratio

$$
\frac{M_{13}}{M_{11}}=P_{33} \frac{A_{3}{ }^{1}}{A_{1}^{1}}=-P_{33} \frac{H_{31}-j\left(\left|H_{12}\right| / H_{12}\right) H_{32}}{H_{33}-H_{11}}
$$

a quantity which must be small in order for this result to hold. In a later section we use this result to discuss sources of noise in beam-type parametric amplifiers.

\section{BEAM DYNAMICS IN COUPLED MODE FORM}

The motion of a filamentary beam of electrons in spatially varying magnetic and electric fields is considered, and the results in terms of cyclotron and synchronous mode amplitudes are expressed. The equations of motion in polarization variables ${ }^{17}$ are

$$
\begin{aligned}
\partial v_{x} / \partial t+v_{z}\left(\partial v_{x} / \partial z\right) & =-\eta\left(E_{x}+v_{y} B_{z}-v_{z} B_{y}\right) \\
\partial v_{y} / \partial t+v_{z}\left(\partial v_{y} / \partial z\right) & =-\eta\left(E_{y}-v_{x} B_{z}+v_{z} B_{x}\right) \\
\partial v_{z} / \partial t+v_{z}\left(\partial v_{z} / \partial z\right) & =-\eta\left(E_{z}+v_{x} B_{y}-v_{y} B_{x}\right) \\
\partial x / \partial t+v_{z}(\partial x / \partial z) & =v_{x} \\
\partial y / \partial t+v_{z}(\partial y / \partial z) & =v_{y} .
\end{aligned}
$$

It is further assumed that the transverse components of the electric and magnetic field vanish at the unperturbed position of the beam (which is taken to be the $z$ axis). In general, these components do not vanish off axis, and for axially symmetric fields near the axis and for quadrupole fields they are linearly proportional to the distance from the axis. Thus in linearizing Eqs. (22a)-(22e), $E_{x}, E_{y}, B_{x}$, and $B_{y}$ are considered as first-order signal quantities. The transverse velocity ( $v_{x}$ and $v_{y}$ ) and position ( $x$ and $y$ ) are first-order quantities. In computing the transverse motion, only the unperturbed part of $v_{z}$ enters and it is easily computed from Eq. (22c) in terms of the known electric field. We subsequently regard $v_{z}$ as a known function of $z$ and $t$, and call it $u_{0}$.

New variables which are simply linear combinations of transverse position and velocity variables are introduced:

$$
\begin{aligned}
& a_{1}=k\left[v_{x}+j v_{y}\right] \\
& a_{2}=k\left[v_{x}-j v_{y}\right] \\
& a_{3}=k\left[v_{x}-j v_{y}+j \omega_{c}(x-j y)\right] \\
& a_{4}=k\left[v_{x}+j v_{y}-j \omega_{c}(x+j y)\right],
\end{aligned}
$$

where $k=\left(I_{0} / 8 \eta \omega_{c}\right)^{\frac{1}{2}}$ and $\omega_{c}=\eta B_{z}(0)$. In general, $B_{z}(0)$ (the value of the axial component of field on the axis), $\omega_{c}$, and $k$ depend on $z$. The preceding variables are therefore defined in terms of the local value of axial magnetic field. When both $\omega_{c}$ and $u_{0}$ are constant and a single frequency is present, $a_{1}, a_{2}, a_{3}$, and $a_{4}$ reduce to the fast cyclotron mode, the slow cyclotron mode, and the two synchronous modes, respectively. When written in terms of the new variables the dynamical equations are

$$
\begin{aligned}
& \left(\frac{d}{d t}-j \omega_{c}\right) a_{1}=-\eta k\left(E_{+}+j u_{0} B_{+}\right)+\frac{1}{k} \frac{d k}{d t} a_{1} \\
& \left(\frac{d}{d t}+j \omega_{c}\right) a_{2}=-\eta k\left(E_{-}-j u_{0} B_{-}\right)+\frac{1}{k} \frac{d k}{d t} a_{2}
\end{aligned}
$$

${ }^{17}$ D. L. Bobroff, I.R.E. Trans. on Electron Devices ED-6, 68 $17 \mathrm{D}$.
(1959) 


$$
\begin{aligned}
& \frac{d a_{3}}{d t}=-\eta k\left(E_{-}-j u_{0} B_{-}\right)+\frac{1 d k}{k} \frac{d t}{d t} a_{3}+\frac{1}{\omega_{c}} \frac{d \omega_{c}}{d t}\left(a_{3}-a_{2}\right) \\
& \frac{d a_{4}}{d t}=-\eta k\left(E_{+}+j u_{0} B_{+}\right)+\frac{1}{k} \frac{d k}{d t} a_{4}+\frac{1}{\omega_{c}} \frac{d \omega_{c}}{d t}\left(a_{4}-a_{1}\right),
\end{aligned}
$$

where $E_{ \pm}=E_{x} \pm j E_{y}$ and $B_{ \pm}=B_{x} \pm j B_{y}$, and $d / d t=(\partial / \partial t)$ $+u_{0}(\partial / \partial z)$. When the magnetic field is constant as in the quadrupole amplifier,

$$
d \omega_{c} / d t=d k / d t=B_{+}=B_{-}=0,
$$

and the equations simplify substantially. We study this case in Sec. III.

\section{Wave Solutions of the Drifting Beam}

For a drifting beam in the absence of transverse electric and magnetic fields, the right-hand sides of Eqs. (24a)-(24d) vanish, and the solutions for sinusoidal time dependence $e^{j \omega t}$ are:

$$
\begin{array}{ll}
a_{1}=A_{1} e^{-j \beta_{1} z} & \beta_{1}=\left(\omega-\omega_{c}\right) / u_{0} \\
a_{2}=A_{2} e^{-j \beta_{2} z} & \beta_{2}=\left(\omega+\omega_{c}\right) / u_{0} \\
a_{3}=A_{3} e^{-j \beta_{3} z} & \beta_{3}=\omega / \mu_{0} \\
a_{4}=A_{4} e^{-j \beta_{4} z} & \beta_{4}=\omega / u_{0} .
\end{array}
$$

If the frequency is positive $(\omega>0)$, these solutions describe the fast and slow cyclotron modes and the two synchronous modes, respectively. In the case of modes $a_{1}$ and $a_{4}$, the beam position $(x, y)$ at a plane of constant $z$ rotates in the same sense as the gyration of electrons in the magnetic field. For modes $a_{2}$ and $a_{3}$ the beam position rotates in the opposite sense. Siegman ${ }^{11}$ has shown that when slow wave circuits with circularly polarized fields are employed, only one pair of modes (either $a_{1}$ and $a_{4}$ or $a_{2}$ and $a_{3}$, depending on the sense of the polarization of the circuit fields) is coupled to the circuit. Modes 2 and 4 carry negative power ${ }^{11,18}\left(P_{22}\right.$ $\left.=P_{44}=-1\right)$, and modes 1 and 3 carry positive power $\left(P_{11}=P_{33}=+1\right)$, and the mode amplitudes have been defined in such a way that their square gives power divided by frequency. Thus

$$
P_{k} / \omega=\left|a_{1}\right|^{2}-\left|a_{2}\right|^{2}+\left|a_{3}\right|^{2}-\left|a_{4}\right|^{2} .
$$

If the frequency is negative $(\omega<0), a_{1}$ describes a slow cyclotron wave instead of a fast cyclotron wave and $a_{2}$ describes a fast cyclotron wave instead of a slow cyclotron wave. Similarly, the roles of $a_{3}$ and $a_{4}$ are interchanged for negative frequencies.

\section{ELECTRIC QUADRUPOLE FIELDS}

This section generalizes the ideas of Adler, Wade, and Gordon and consider the action of a traveling quadrupole

18 C. C. Johnson, J. Appl. Phys. 31, 338 (1960).

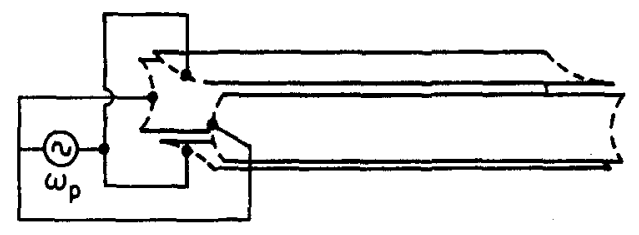

(a)

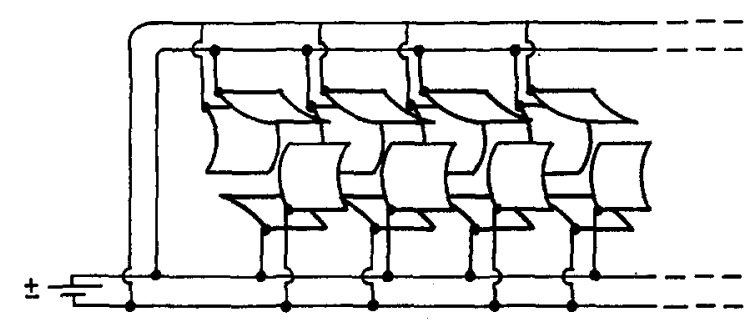

(b)

FIG. 1. Quadrupole pump structures: (a) $\beta_{p}=0$, (b) $\omega_{p}=0$.

pump field whose potential near the axis is given by

$$
\phi(r, \theta, z, t)=V_{p}(r / a)^{2} \cos 2 \theta \cos \left(\omega_{p} b-\beta_{p} z\right) .
$$

The means for producing a pump field of this sort is shown in Fig. 1 for two special cases: (a) $\beta_{p}=0$; (b) $\omega_{p}=0$. The latter case corresponds to Gordon's proposal for dc pumping. ${ }^{2}$ Noting that $r^{2} \cos 2 \theta=x^{2}-y^{2}$, the electric fields derived from Eq. (26) are

$$
\begin{aligned}
& E_{x}=-2\left(V_{p} / a^{2}\right) x \cos \left(\omega_{p} t-\beta_{p} z\right) \\
& E_{y}=+2\left(V_{p} / a^{2}\right) y \cos \left(\omega_{p} t-\beta_{p} z\right) .
\end{aligned}
$$

When these are substituted into Eqs. (24a)-(24d), a set of four partial differential equations with coefficients which depend on $t$ and $z$ results:

$$
\begin{aligned}
& {\left[\frac{\partial}{d t}+u_{0} \frac{\partial}{\partial z}-j \omega_{c}\right] a_{1}=-2 j-\underset{\omega_{c}}{\Omega^{2}} \cos \left(\omega_{p} t-\beta_{p} z\right)\left[a_{3}-a_{2}\right]} \\
& {\left[\frac{\partial}{d t}+u_{0} \frac{\partial}{\partial z}+j \omega_{c}\right] a_{2}=2 \underset{\omega_{c}}{\Omega^{2}} \cos \left(\omega_{p} t-\beta_{p} z\right)\left[a_{4}-a_{1}\right]}
\end{aligned}
$$

$$
\begin{aligned}
& {\left[\frac{\partial}{\partial t}+u_{0} \frac{\partial}{\partial z}\right] a_{3}=\underset{\omega_{c}}{2 j-} \cos \left(\omega_{p} t-\beta_{p} z\right)\left[a_{4}-a_{1}\right]} \\
& {\left[\frac{\partial}{\partial t}+u_{0} \frac{\partial}{\partial z}\right] a_{4}=-\underset{\omega_{c}}{2 j-} \cos \left(\omega_{p} t-\beta_{p} z\right)\left[a_{3}-a_{2}\right] \text {, }}
\end{aligned}
$$

where the magnetic field is assumed to be constant and $\Omega^{2} \equiv|e| V_{p} / m a^{2}$ is a pump strength parameter which has the dimensions of frequency squared. It is assumed here that the pump structure will support only the pump field [Eq. (26)] and that it will support no waves which have the same frequency and propagation constant as any of the beam modes. 
Equations (28) must be solved subject to the boundary conditions that $a_{1}, a_{2}, a_{3}$, and $a_{4}$ are known functions of time on an initial plane $z=0$. The simplest case of interest is one in which a single mode has sinusoidal time dependence $e^{j \omega t}$ at the initial plane. In the absence of the pump $\Omega^{2}=0$, the appropriate equation from Eqs. (28a)-(28d) shows that the mode would simply propagate down the beam $a_{i} \sim e^{-j \beta z}$. When the pump is present, however, this mode also acts as a source for other modes by virtue of the additional terms on the right-hand side of Eqs. (28). When a mode amplitude proportional to $e^{j(\omega t-\beta z)}$ is multiplied by the pump quantity $\cos \left(\omega_{p} t-\beta_{p} z\right)$, two new terms of the form $e^{j\left(\omega_{n} t-\beta_{n} z\right)}$ are produced, where

$$
\begin{aligned}
& \omega_{1}=\omega+\omega_{p} \quad \beta_{1}=\beta+\beta_{p} \\
& \omega_{-1}=\omega-\omega_{p} \quad \beta_{-1}=\beta-\beta_{p} .
\end{aligned}
$$

It may be said that the new terms have their frequency and propagation constant shifted by $\pm \omega_{p}$ and $\pm \beta_{p}$, respectively, from the original wave. This is shown schematically in the $\omega-\beta$ diagram of Fig. 2 . Not only does a wave $(\omega, \beta)$ lead to the new terms $\left(\omega_{1}, \beta_{1}\right)$ and $\left(\omega_{-1}, \beta_{-1}\right)$, but each new term leads to additional new terms so that the entire set of waves $\left(\omega_{n}=\omega+n \omega_{p}\right.$, $\beta_{n}=\beta+n \beta_{p}$ ), where $n$ is any positive or negative integer, is present.

Solutions will be of the form

$$
a_{i}=\sum_{n} a_{i, n}(z) e^{j\left(\omega_{n} t-n \beta_{p z}\right)},
$$

where, for generality, we have allowed for the possibility that each idler has a different $z$ dependence. In order to obtain a set of constant coefficient equations for the amplitudes $a_{i, n}(z)$ we also have factored part of the $z$ dependence, $e^{-i n \beta_{p} z}$. Upon substituting Eq. (29) into Eq. 28 and making use of the orthogonality of different frequency components, one obtains equations for the signal and idler amplitudes in coupled mode form

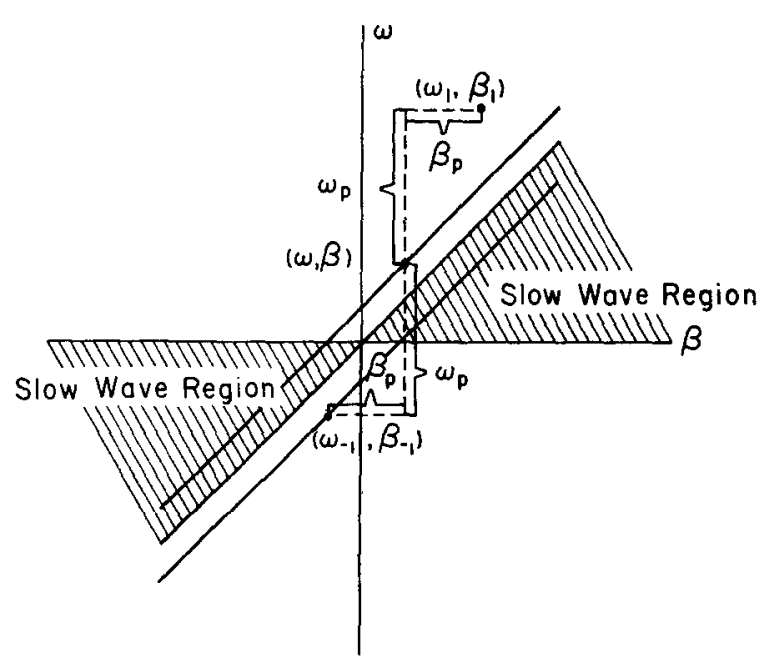

FIG. 2. $\omega-\beta$ diagram illustrating the generation of new modes by the pump.

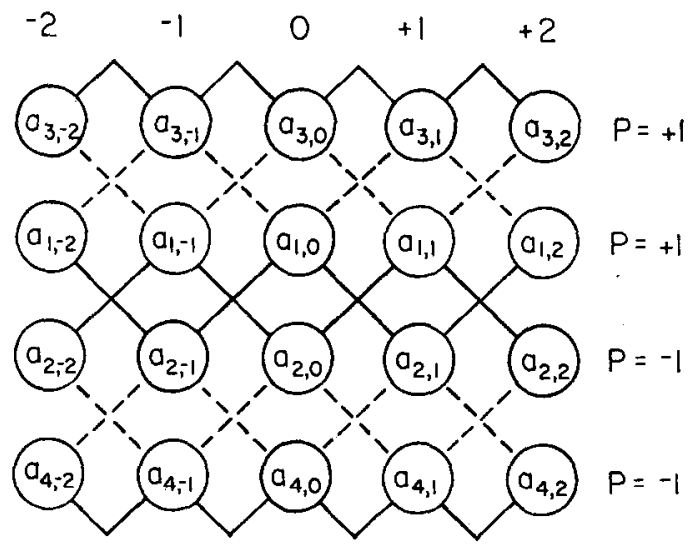

FIG. 3. Diagram showing coupling of modes in a quadrupole pump field. Solid lines indicate active coupling of two modes and dashed lines indicate passive coupling. The lines from $a_{q}$ at the bottom are to be imagined as connected to the lines from $a_{3}$ at the top by wrapping the diagram into a cylinder.

[compare with Eq. (1)]:

$$
\begin{aligned}
& {\left[-j \frac{\partial}{\partial z}+\frac{\left(\omega_{n}-\omega_{c}-n \beta_{p} u_{0}\right)}{u_{0}}\right] a_{1, n}} \\
& \quad+\frac{\Omega^{2}}{\omega_{c} u_{0}}\left[a_{3, n-1}-a_{2, n-1}+a_{3, n+1}-a_{2, n+1}\right]=0
\end{aligned}
$$

$$
\left[-j \frac{\partial}{\partial z}+\frac{\left(\omega_{n}+\omega_{c}-n \beta_{p} u_{0}\right)}{u_{0}}\right] a_{2, n}
$$

$$
-\frac{\Omega^{2}}{\omega_{c} u_{0}}\left[a_{4, n-1}-a_{1, n-1}+a_{4, n+1}-a_{1, n+1}\right]=0 ;
$$

$$
\left[-j \frac{\partial}{\partial z}+\frac{\omega_{n}-n \beta_{p} u_{0}}{u_{0}}\right] a_{3, n}
$$

$$
\begin{aligned}
& -\frac{\Omega^{2}}{\omega_{c} u_{0}}\left[a_{4, n-1}-a_{1, n-1}+a_{4, n+1}-a_{1, n-1}\right]=0 ; \\
& {\left[-j \frac{\partial}{\partial z}+\frac{\omega_{n}-n \beta_{p} u_{0}}{u_{0}}\right] a_{4, n}} \\
& +\frac{\Omega^{2}}{\omega_{c} u_{0}}\left[a_{3, n-1}-a_{2, n-1}+a_{3, n+1}-a_{2, n+1}\right]=0 .
\end{aligned}
$$

It is convenient to interpret these equations as describing the propagation of a set of coupled modes. The uncoupled modes are simply those modes which can exist on the beam at signal and idler frequencies $\left(\omega_{n}\right)$ in in the absence of a pump (see Sec. II). The effect of the pump is to couple these modes together. Figure 3 has been constructed from Eqs. (30a)-(30d) and shows diagrammatically which modes are coupled together. A solid line drawn between two modes indicates active coupling leading to the possibility of growth, while a 
dashed line indicates passive coupling. Parity assignments of modes implied by Eqs. (30a)-(30d) are also shown. $a_{3, n}$ and $a_{1, n}$ have positive parity, and $a_{2, n}$ and $a_{4, n}$ have negative parity.

It is seen that $a_{1,0}$ is directly coupled to $a_{3,-1}, a_{2,-1}$, $a_{3,1}$, and $a_{2,1}$. When any one of the latter uncoupled modes has approximately the same $z$ dependence as that of $a_{1,0}$, the effect of such a coupling is strong. The $z$ dependences of the uncoupled modes are obtained from the diagonal elements $H_{i i}$ of Eqs. (30a) $-(30 \mathrm{~d})$ :

$$
\frac{\omega-\omega_{c}+n \Omega_{p}}{u_{0}} \frac{\omega+\omega_{c}+n \Omega_{p}}{u_{0}} \frac{\omega+n \Omega_{p}}{u_{0}} \frac{\omega+n \Omega_{p}}{u_{0}},
$$

where $\Omega_{p}=\omega_{p}-\beta_{p} u_{0}$ is the Doppler-shifted pump frequency. Figure 4 shows a plot of $\omega$ vs $H_{i i}$ for the various uncoupled modes. ${ }^{19}$ Each set of curves is displaced horizontally from its neighbors by an amount $\Omega_{p} / u_{0}$.

\section{Twisted Quadrupole}

Before discussing the solution and interpretation of these equations we also quote similar equations applying to a twisted quadrupole pump field, ${ }^{20}$ whose potential near the axis is given by

$$
\phi(r, \theta, z, t)=\frac{1}{2} V_{p}(r / a)^{2} \cos \left(\omega_{p} t-\beta_{p} z \pm 2 \theta\right) .
$$

Such a potential may be generated by means of an octufilar helix driven as shown in Fig. 5. Note that for

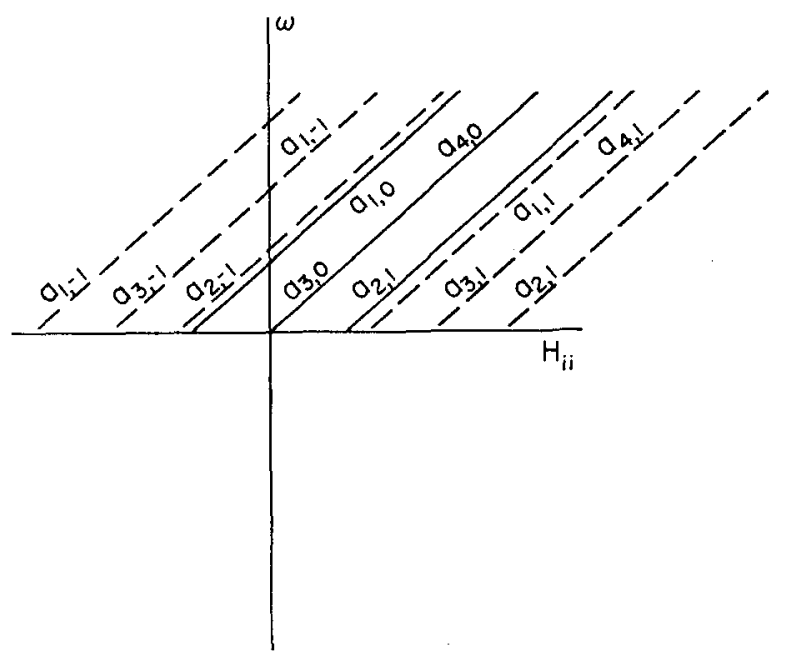

FIG. 4. $\omega$ vs $\Pi_{i i}$ of the uncoupled modes.

${ }_{19}$ We have refrained from calling $H_{i i}$ the propagation constant of the $i$ th uncoupled mode because we have already factored part of the $z$ dependence. When this $z$ dependence is reinserted, we find the uncoupled mode propagation constants to be

$$
\left(\omega_{n}-\omega_{c} / u_{0}\right)\left(\omega_{n}+\omega_{c} / u_{0}\right)\left(\omega_{n} / u_{0}\right)\left(\omega_{n} / u_{0}\right)
$$

when the frequency is $\omega_{n}$, i.e., the uncoupled modes are just fast and slow cyclotron and synchronous waves at frequency $\omega_{n}$ when $\omega_{n}>0$, and when $\omega_{n}<0$ the role of fast and slow waves is interchanged.

20 The special case of dc pumping $\left(\omega_{p}=0\right)$ with a quadrufilar helix is discussed by A. E. Siegman, Proc. I.R.E. 48, 1750 (1960).

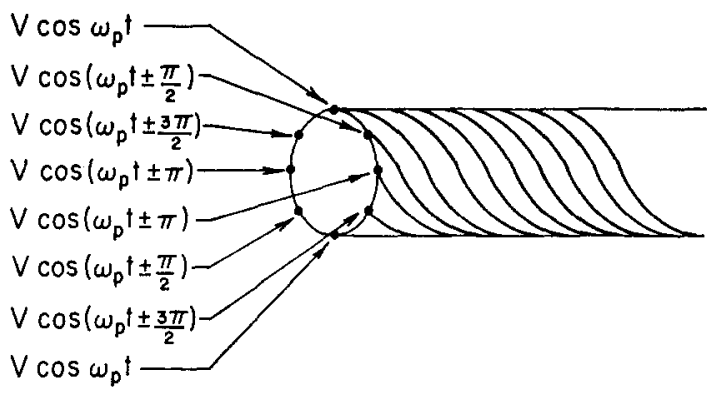

FIG. 5. Twisted octufilar helix which gives rise to the potential given by Eq. (32).

$\omega_{p}=0$, only four wires are necessary to create the twisted quadrufilar field, since the other wires are at zero potential.

When the pump fields derived from this potential are substituted into Eqs. (18a)-(18d), one obtains equations analogous to $(24 a)-(24 d)$ :

$$
\begin{aligned}
{\left[-j \frac{\partial}{\partial z}+\frac{\left(\omega_{n}-\omega_{c}-n \beta_{p} u_{0}\right)}{u_{0}}\right.} & ] a_{1, n} \\
& -\frac{\Omega^{2}}{\omega_{c} u_{0}}\left[a_{2, n \pm 1}-a_{3, n \pm 1}\right]=0
\end{aligned}
$$

$\left[-j \frac{\partial}{\partial z}+\frac{\left(\omega_{n}+\omega_{c}-n \beta_{p} u_{0}\right)}{u_{0}}\right] a_{2, n}$

$$
\begin{array}{r}
-\frac{\Omega^{2}}{\omega_{c} u_{0}}\left[a_{4, n \uparrow 1}-a_{1, n \uparrow 1}\right]=0 ; \\
{\left[-j \frac{\partial}{\partial z}+\frac{\omega_{n}-\beta_{p} u_{0}}{u_{0}}\right] a_{3, n}-\frac{\Omega^{2}}{\omega_{c} u_{0}}\left[a_{4, n \mp 1}-a_{1, n \mp 1}\right]=0 ;} \\
{\left[-j \frac{\partial}{\partial z}+\frac{\omega_{n}-\beta_{p} u_{0}}{u_{0}}\right] a_{4, n}-\frac{\Omega^{2}}{\omega_{c} u_{0}}\left[a_{2, n \pm 1}-a_{3, n \pm 1}\right]=0 .}
\end{array}
$$

Comparison with Eqs. (30a)-(30d) shows that, with the twisted quadrupole, modes of a particular frequency ( $n$ value) are coupled either to modes of the next higher frequency or to modes of the next lower frequency, but not to both. This feature, illustrated schematically in Fig. 6(a) and (b), is seen to be inherently simpler than that of the previous example, since only modes with the proper polarization are coupled by the pump field.

\section{Quadrupole Solutions}

By virtue of the time- and space-dependent pump wave, modes whose frequencies differ by $\pm \omega_{p}$ and whose propagation constants differ by about $\pm \beta_{p}$ are coupled together. In order for a strong interaction between two modes to occur, two conditions must be fulfilled: 
R. W. GOULD AND C. C. JOHNSON

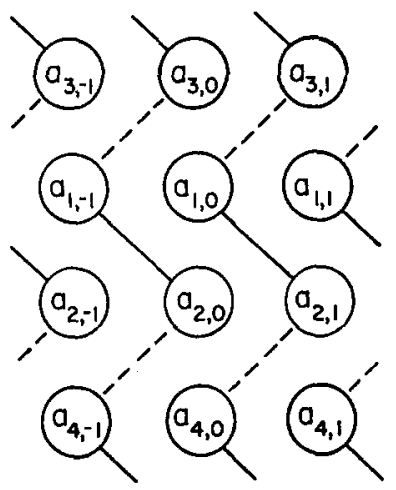

Upper Sign in (32)

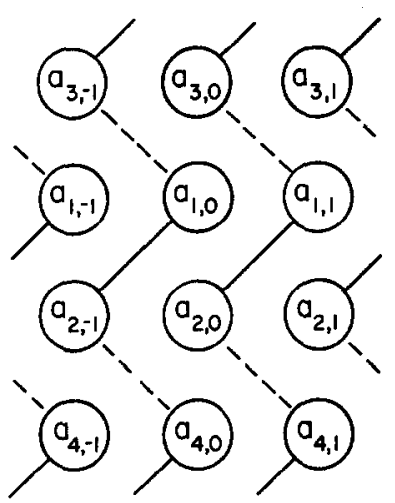

Lower Sign in (32)

(a) Direct coupling between the modes must exist (see Fig. 3 for summary of the couplings);

(b) The modes must have nearly the same diagonal elements $H_{i i}$, i.e., the corresponding lines in Fig. 4 must nearly overlap.

For the case of the twisted quadrupole, there are three different values of Doppler-shifted pump frequency for each choice of sign in Eq. (32) for which strong interaction can occur. Figure 7 shows the modes which are strongly coupled (same values of $H_{i i}$ ) and summarizes the conditions for which strong interaction occurs. When the pump potential is given by Eq. (26) instead of Eq. (32), all of the strong interactions shown in Fig. 7 are also possible, since Eq. (26) contains terms corresponding to both choices of sign in Eq. (32).

For either type of quadrupole field, the strong coupling between $a_{1,0}$ and $a_{2,-1}$, which arises when $\Omega_{p}=2 \omega_{c}$, leads to amplification. $a_{1,0}$ is a fast cyclotron wave and if $\omega_{p}>\omega$ (high-frequency pumping ${ }^{1}$ ), $a_{2,-1}$ is also fast cyclotron wave (see Fig. 2). Whereas if $\omega_{p}<\omega$ (low-frequency or dc pumping ${ }^{2}$ ), $a_{2,-1}$ is a slow cyclotron wave. Regardless of the pump frequency, the two modes have opposite parity and a growing and decaying pair of waves results. We note also that the slow cyclotron wave $a_{2,0}$ is coupled to a fast cyclotron wave $a_{1,1}$, whose frequency is $\omega+\omega_{p}$, producing a growing and decaying pair of waves.

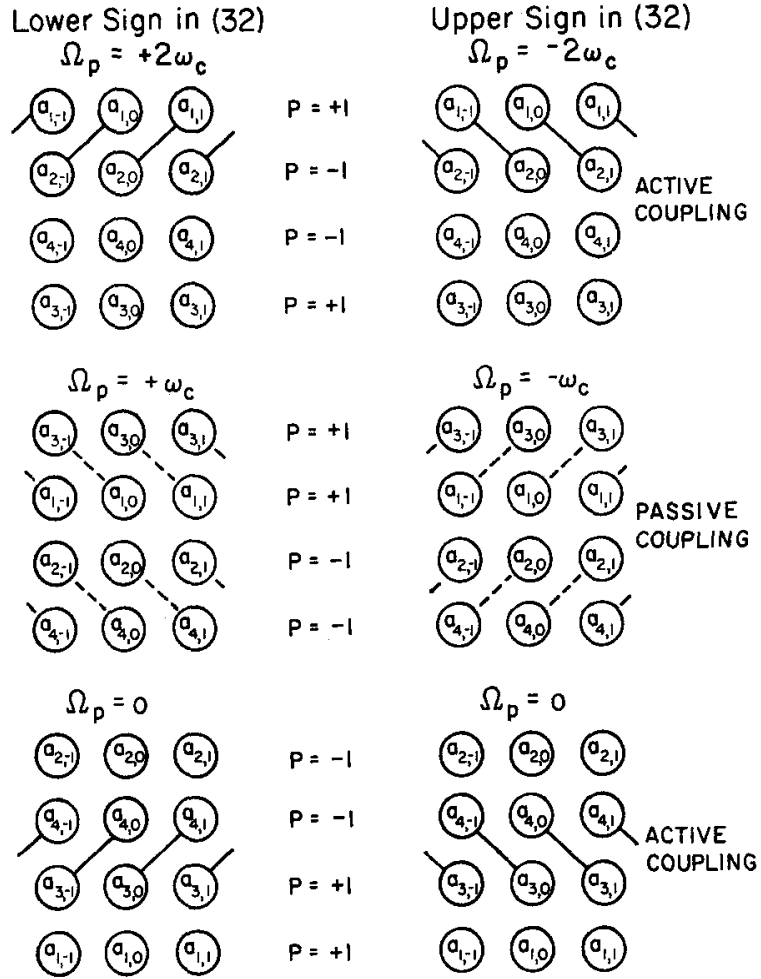

FIG. 7. Diagram showing which modes are strongly interacting in a twisted electric quadrupole field.

The rates of growth and decay are easily obtained by neglecting all but the two modes which have nearly the propagation constants. The effect of the weakly interacting synchronous modes is discussed in the next section. Taking $a_{1,0}$ and $a_{2,-1}$ to be the first and second modes, respectively, we have

$$
\begin{array}{ll}
H_{11}=\left(\omega-\omega_{c}\right) / u_{0} & H_{12}=-\left(\Omega^{2} / \omega_{c} u_{0}\right) \\
H_{21}=\Omega^{2} / \omega_{c} u_{0} & H_{22}=\left(\omega+\omega_{c}-\Omega_{p}\right) / u_{0} .
\end{array}
$$

On substituting into Eq. (15),

$$
\beta u_{0}=\left(\omega-\omega_{c}-\frac{1}{2} \Delta \Omega_{p}\right) \pm\left[\left(\frac{1}{2} \Delta \Omega_{p}\right)^{2}-\left(\Omega^{2} / \omega_{c}\right)\right]^{\frac{1}{3}},
$$

where $\Delta \Omega_{p}=\Omega_{p}-2 \omega_{c}$ is the difference between the Doppler-shifted pump frequency and $2 \omega_{c}$. Growth is possible if $\left(\Delta \Omega_{p}\right)<2 \Omega^{2} / \omega_{c}$. The weaker the pump (small $\Omega^{2}$ ) the more nearly equal the Doppler-shifted pump frequency and twice the cyclotron frequency must be in order to achieve growth. When $\Delta \Omega_{p}=0$,

$$
\beta=\left(\omega-\omega_{c}\right) / u_{0} \pm j\left(\Omega^{2} / \omega_{c} u_{0}\right) .
$$

The spatial rate of growth $\Omega^{2} / \omega_{c} u_{0}$ is proportional to the pump strength. This result is the same for both highfrequency and for low-frequency pumping.

A similar analysis of the coupling between $a_{2,0}$, a slow cyclotron wave at frequency $\omega$; and $a_{1,1}$, a fast cyclotron wave at frequency $\omega+\omega_{p}$, yields a growing and decaying pair of waves with exactly the same growth rate and

$$
\beta_{u_{0}}=\left(\omega+\omega_{c}-\frac{1}{2} \Delta \Omega_{p} \pm\left[\left(\frac{1}{2} \Delta \Omega_{p}\right)^{2}-\left(\Omega^{2} / \omega_{c}\right)^{2}\right]^{\frac{1}{2}} .\right.
$$




\section{Source of Noise in the Quadrupole Amplifier}

Figure 3 and Eqs. (30a)-(30d) show that six other weakly interacting modes, $a_{3,-1} a_{3,1}, a_{2,1}, a_{4,-2}, a_{1,-2}$, and $a_{4,0}$, are also coupled directly to the strongly interacting pair of modes which give rise to gain, ${ }^{21} a_{1,0}$, and $a_{2,-1}$. In general, these modes will not be stripped of noise, and we can evaluate their contribution to the output noise temperature with the aid of Eqs. (21) and (30). Table I shows the values of $M_{1 i} / M_{11}(i=3,4,5,6,7,8)$ so obtained for $\Delta \Omega_{p}=0$. By inserting the transverse velocities emanating from a hot cathode ${ }^{22}$ into the mode definitions [Eqs. (23)], the noise power for each of these weakly coupled modes can be found. Adler and $\mathrm{Wade}^{23}$ have shown by this procedure that the noise power carried by a cyclotron wave is $\left(\omega / \omega_{c}\right) k T_{c} \Delta f$. The synchronous waves will carry noise power from the transverse displacements in addition to that from the transverse velocities, as seen from Eqs. (23c) and (23d). This additional term can easily be shown to be small when the cathode radius is small compared to the Larmor radius $\left(k T_{c} / m\right)^{\frac{1}{2}} / \omega_{c}{ }^{22}$ The minimum noise carried by all the weakly coupled modes can be set at $\left(\omega / \omega_{c}\right) k T_{c} \Delta f$, and the noise temperature of the device from these sources is $2.70 \xi^{2} T_{\text {eathode }}$ in the Adler-Wade tube. Most of the noise comes from the $a_{4,0}$ and $a_{3,-1}$ synchronous modes at the signal and idler frequencies, respectively. Equation (36) shows that $\xi$ is just $1 / 2 \pi$ times the gain in nepers per cyclotron wavelength. Hence the gain per cyclotron wavelength should be small to minimize this source of noise. In the Adler quadrupole amplifier $\xi \cong \frac{1}{8}$, and noise contributed from these sources is estimated to be of the order of $40^{\circ} \mathrm{K}$. An accurate calculation would require a detailed knowledge of the noise of the various weakly interacting modes, and hence a theory of the gun region.

Note added in proof. R. Adler has informed us that the measured noise appears to be independent of the pump strength. This would rule out the explanation, described here, of the noise observed in quadrupole amplifiers.

We also note from Fig. 6(b) or from Eqs. (33a)-(33d) that, if a twisted quadrupole is employed, the two major source of noise, $a_{4,0}$ and $a_{3,-1}$, are still present although the other modes, $a_{3,1}, a_{2,1}, a_{4,-2}$, and $a_{1,-2}$, are no longer coupled.

\section{Parametric Refrigeration}

Sturrock has recently proposed a method for removing noise from the negative energy slow space-charge wave. ${ }^{24}$ The present paper now shows how this idea can be extended to make possible the removal of noise from the negative energy slow cyclotron wave. ${ }^{25} \mathrm{~A}$ require-

\footnotetext{
${ }^{21}$ Other modes are coupled indirectly to $a_{1,0}$ and $a_{2,-1}$ through the modes we are considering, but they make an even smaller contribution to the output noise.

${ }_{22}$ G. Wade, K. Amo, and D. A. Watkins, J. Appl. Phys. 25, 1514 (1954).

${ }^{23}$ R. Adler and G. Wade, J. Appl. Phys. 31, 1261 (1960).

${ }_{24}$ P. A. Sturrock, M. L. Rept. No. 656, Stanford University, Stanford, California (October, 1959).

${ }_{25}$ The method described below has been proposed independently, and verified experimentally, by E. I. Gordon and A. Ashkin (to be published).
}

ment for such a scheme is that we have passive coupling between the negative energy slow cyclotron wave and a positive energy wave from which the noise can be removed by conventional means. This is only possible if the latter is a negative frequency wave, i.e., if highfrequency pumping $\left(\omega_{p}>\omega\right)$ is employed. Such a coupling exists between $a_{2,0}$ and $a_{4,-1}$, according to Fig. 7 , and we must arrange the pump circuit so that $\Omega_{p}=\omega_{p}-\beta_{p} u_{0}=-\omega_{c}$. If a twisted quadrupole is employed, the potential must correspond to the upper sign in Eq. (32), i.e., the pitch of the helix must have the same sense as the spiral orbit of an electron in the uniform field. The strength of the pump field for complete energy interchange in a distance $L$ is determined by the condition ${ }^{26}$

$$
\left(\Omega^{2} / \omega_{c} u_{0}\right) L=\pi / 2 .
$$

The noise on the synchronous idler mode $a_{4,-1}$ must first be removed by allowing it to interact with a slow wave circuit.

The slow synchronous mode $a_{40}$ could be cooled, in a similar manner, by coupling it passively to a fast cyclotron idler $a_{2-1}$ which has previously been cooled with a Cuccia coupler. ${ }^{27}$ The pump requirement is $\Omega_{p}=\omega_{p}-\beta_{p} u_{0}=+\omega_{c}$, and the upper sign in Eq. (32) is appropriate.

\section{AXIALLY SYMMETRIC ELECTRIC AND MAGNETIC FIELDS}

When the electric and magnetic fields are axially symmetric, the transverse components near the axis are related to longitudinal components on the axis (which we label simply $B$ and $E$ in this discussion):

$$
\begin{aligned}
& B_{x} \cong-\frac{1}{2} B^{\prime} x \quad B_{y} \cong-\frac{1}{2} B^{\prime} y \\
& E_{x} \cong-\frac{1}{2} E^{\prime} x \quad E_{y} \cong-\frac{1}{2} E^{\prime} y,
\end{aligned}
$$

where the prime denoted differentiation with respect to $z(\partial / \partial z)$. These relations are valid even when $E$ and $B$ depend on time, since they follow directly from $\nabla \cdot \mathbf{E}$ $=\nabla \cdot \mathbf{B}=0$. Substituting Eqs. (39a) and (39b) into Eqs. (24a) $-(24 \mathrm{~d})$, and using Eqs. (23a) $-(23 \mathrm{~d})$ to express $x$ and $y$ in terms of $a_{1}, a_{2}, a_{3}$, and $a_{4}$, one obtains:

$$
\begin{aligned}
\left(\frac{\partial}{\partial t}+u_{0} \frac{\partial}{\partial z}-j \omega_{c}\right) a_{1} & =-\frac{u_{0} B^{\prime}}{2 B} a_{4}+j \frac{E^{\prime}}{2 B}\left(a_{4}-a_{1}\right) \\
\left(\frac{\partial}{\partial t}+u_{0} \frac{\partial}{\partial z}+j \omega_{c}\right) a_{2} & =-\frac{u_{0} B^{\prime}}{2 B} a_{3}-j \frac{E^{\prime}}{2 B}\left(a_{3}-a_{2}\right) \\
\left(\frac{\partial}{\partial t}+u_{0} \frac{\partial}{\partial z}\right) a_{3} & =-\frac{u_{0} B^{\prime}}{2 B} a_{2}-j \frac{E^{\prime}}{2 B}\left(a_{3}-a_{2}\right) \\
\left(\frac{\partial}{\partial t}+u_{0} \frac{\partial}{\partial z}\right) a_{4} & =-\frac{u_{0} B^{\prime}}{2 B} a_{1}+j \frac{E^{\prime}}{2 B}\left(a_{4}-a_{1}\right) .
\end{aligned}
$$

${ }^{26}$ Because the coupling between any pair of modes involves the same coefficient, this is the condition which must exactly hold for complex interchange between any pair of strongly interacting passively coupled modes.

${ }_{27}$ C. L. Cuccia, RCA Rev. 10, 270 (1949). 
It must be remembered that in general $u_{0}$ and $\omega_{\text {r are }}$ also functions of $z$ and $t$.

\section{Adiabatic Changes}

If $\left|H_{i j} H_{j i}\right| \ll\left|H_{i i}-H_{j j}\right|^{2}$, we may neglect coupling between modes $i$ and $j$. If the magnetic and electric fields change sufficiently slowly, i.e., if $u_{0} B^{\prime} / 2 B$ and $E^{\prime} / 2 B$ are small compared with $\omega_{c}$, Eqs. (40a) and (40b) become

$$
\begin{array}{r}
{\left[-j(\partial / \partial z)+\left(\omega-\omega_{c}+E^{\prime} / 2 B\right) / u_{0}\right] a_{1} \cong 0} \\
{\left[-j(\partial / \partial z)+\left(\omega+\omega_{c}-E^{\prime} / 2 B\right) / u_{0}\right] a_{2} \cong 0} \\
{\left[-j(\partial / \partial z)+\left(\omega+E^{\prime} / 2 B\right) / u_{0}\right] a_{3} \cong 0} \\
{\left[-j(\partial / \partial z)+\left(\omega-E^{\prime} / 2 B\right) / u_{0}\right] a_{4} \cong 0,}
\end{array}
$$

where sinusoidal time dependence has been assumed. These equations have as their solutions

$$
\begin{aligned}
& a_{1}(z)=a_{1}(0) \exp \left[-j \int_{0}^{z} d z\left(\omega-\omega_{c}+E^{\prime} />B\right) / u_{0}\right] \\
& a_{2}(z)=a_{2}(0) \exp \left[-j \int_{0}^{z} d z\left(\omega+\omega_{c}-E^{\prime} / 2 B\right) / u_{0}\right] \\
& a_{3}(z)=a_{3}(0) \exp \left[-j \int_{0}^{z} d z\left(\omega+E^{\prime} / 2 B\right) / u_{0}\right] \\
& a_{4}(z)=a_{4}(0) \exp \left[-j \int_{0}^{z} d z\left(\omega-E^{\prime} / 2 B\right) / u_{0}\right]
\end{aligned}
$$

The mode amplitudes remain constant, aside from a phase factor, during an adiabatic increase or decrease in electric or magnetic field. Since the modes have been defined in terms of the local magnetic field, the kinetic power of each mode is constant. These results are directly related to the well-known behavior of single particles in slowly varying magnetic fields. ${ }^{28,29}$ The orbit expands or contracts in such a way that $m\left(v_{x}{ }^{2}+v_{y}{ }^{2}\right) / 2 B$, the magnetic moment, remains constant and the radius of the guiding center $R$ moves along a field line, i.e., $B R^{2}$ is constant. The position of the guiding center is $\mathbf{R}=\mathbf{r}-\left(\boldsymbol{\omega}_{c} \times \mathbf{v}\right) / \boldsymbol{\omega}_{c}{ }^{2}$, and according to Eqs. (23a) $-(23 \mathrm{~d})$,

$$
\begin{aligned}
\left(v_{x}{ }^{2}+v_{y}{ }^{2}\right) / B & \sim a_{1}{ }^{2}+a_{2}{ }^{2} \\
R^{2} B & \sim a_{3}{ }^{2}+a_{4}{ }^{2},
\end{aligned}
$$

both of which are constants of the motion.

We note also that, although $E^{\prime} / 2 B u_{0}$ is small, its integral with respect to $z$ may not be, and the phase shift of the two synchronous modes will not always be the same.

\section{Abrupt Jumps}

When a change in the magnetic field takes place in a small fraction of a cyclotron wavelength, all terms in

${ }^{28}$ L. Spitzer, Jr., Physics of Fully Ionized Gases (Interscience Publishers, Inc., New York, 1956), p. 3.

${ }^{29}$ E. I. Gordon, J. Appl. Phys. 31, 1187 (1960).
Eqs. (40a)-(40d) except those involving $b^{\prime}$ and $\partial / \partial z$ may be neglected, and we write:

$$
\begin{aligned}
& \left(d a_{1} / d \xi\right)+a_{4}=0 \\
& \left(d a_{4} / d \xi\right)+a_{1}=0 \\
& \left(d a_{2} / d \xi\right)+a_{3}=0 \\
& \left(d a_{3} / d \xi\right)+a_{2}=0
\end{aligned}
$$

where $d \xi=\left(B^{\prime} / 2 B\right) d z$. Modes 1 and 4 become coupled, as do modes 2 and 3 . These equations are readily solved, and the matrix connecting the mode amplitudes immediatcly following the jump to those immediately preceding the jump has the elements

$$
\begin{aligned}
M_{11}=M_{22}=M_{33} & =M_{44}=\frac{1}{2}\left[\left(B_{2} / B_{1}\right)^{\frac{1}{2}}+\left(B_{1} / B_{2}\right)^{\frac{1}{2}}\right] \\
M_{14}=M_{41}=M_{23}= & M_{32} \\
& =-\frac{1}{2}\left[\left(B_{2} / B_{1}\right)^{\frac{1}{2}}-\left(B_{1} / B_{2}\right)^{\frac{1}{2}}\right]
\end{aligned}
$$

where $B_{1}$ and $B_{2}$ are the values of the magnetic field before and after the jump, respectively.

When a change in potential takes place in a small fraction of a cyclotron wavelength, all terms in Eqs. (40a)-(40d) except those involving $E^{\prime}$ and $\partial / \partial z$ may be neglected, and we write:

$$
\begin{aligned}
& \left(\partial a_{1} / \partial \xi^{\prime}\right)+j\left(a_{1}-a_{4}\right)=0 \\
& \left(\partial a_{4} / \partial \xi^{\prime}\right)+j\left(a_{1}-a_{4}\right)=0 \\
& \left(\partial a_{2} / \partial \xi^{\prime}\right)+j\left(a_{3}-a_{2}\right)=0 \\
& \left(\partial a_{3} / \partial \xi^{\prime}\right)+j\left(a_{3}-a_{2}\right)=0,
\end{aligned}
$$

where $d \xi^{\prime}=\left(E^{\prime} / 2 u_{0} B\right) d z$; as in the case of a jump in magnetic field, mode 1 is coupled to mode 4 and mode 2 is coupled to mode 3 . The matrix elements for the jump are

$$
\begin{aligned}
& M_{11}=M_{33}=1+j \xi^{\prime} \\
& M_{22}=M_{44}=1-j \xi^{\prime} \\
& M_{14}=-M_{41}=M_{32}=-M_{32}=-j \xi^{\prime},
\end{aligned}
$$

where

$$
\xi^{\prime}=\int_{z_{1}}^{z_{2}}\left(E^{\prime} d z / 2 B u_{0}\right)
$$

Making use of the fact that $E^{\prime}=-V^{\prime \prime}$ and $u_{0}=(2 \eta V)^{\frac{1}{2}}$, where $V$ is the potential on the axis, we can express $\xi^{\prime}$ in terms of the focal length of the lens $f_{2}$ :

$$
\xi=-\frac{1}{2 B} \int_{z_{1}}^{z_{2}} \frac{V^{\prime \prime} d z}{(2 \eta V)^{\frac{1}{2}}}=-\frac{u_{02}}{\omega_{c} f_{2}}=\frac{1 \lambda_{e 2}}{2 \pi f_{2}},
$$

where $u_{02}$ and $\lambda_{c 2}$ are the beam velocity and cyclotron wavelength after the lens, respectively. We see that unless the cyclotron wavelength is greater than the focal length there is actually little coupling between modes by an electrostatic lens. 


\section{Periodic Electric Fields}

We now investigate the coupling between modes which is caused by a traveling electric field

$$
E=E_{p} \sin \left(\omega_{p} t-\beta_{p} z\right)
$$

where the magnetic field is constant $\left(B^{\prime}=0\right)$. In such an electric field the $z$ velocity is not constant, but is given by

$$
u_{0}=u_{00}+\left(\eta E_{p} / \Omega_{p}\right) \cos \left(\omega_{p} b-\beta_{p} z\right)
$$

where $u_{00}$ is a constant and $\Omega_{p}=\omega_{p}-\beta_{p} u_{0}$. As before, solutions will have the form given by Eq. (29). When we restrict ourselves to weak fields $\left(\eta E_{p} / \Omega_{p} \ll u_{00}\right)$, we obtain for the coupled mode equations

$$
\begin{aligned}
& {\left[-j \frac{\partial}{\partial z}+\frac{\omega-\omega_{c}+n \Omega_{p}}{u_{00}}\right] a_{i, n}} \\
& =-\frac{\Omega^{\prime 2}}{\omega_{c} u_{00}}\left[a_{4, n+1}+a_{4, n-1}-a_{1, n+1}-a_{1, n-1}\right] \\
& \left.+\frac{2 \Omega^{\prime 2}}{\Omega_{p} u_{00}} \mid \frac{\omega_{n+1}-\omega_{c}}{\beta_{p} u_{00}}-a_{1, n+1}+\frac{\omega_{n-1}-\omega_{c}}{\beta_{p} u_{00}} a_{1, n-1}\right] \\
& {\left[-j \frac{\partial}{\partial z}+\frac{\omega+\omega_{c}+n \Omega_{p}}{u_{00}}\right] a_{2, n}} \\
& =\frac{\Omega^{\prime 2}}{\omega_{c} u_{00}}\left[a_{3, n+1}+a_{3, n-1}-a_{2, n+1}-a_{2, n-1}\right] \\
& +\frac{2 \Omega^{\prime 2}}{\Omega_{p} u_{00}}\left[\frac{\omega_{n+1}+\omega_{c}}{\beta_{p} u_{00}} a_{2, n+1}+\frac{\omega_{n-1}+\omega_{c}}{\beta_{p} u_{00}} a_{2 n-1}\right] \\
& {\left[-j \frac{\partial}{\partial z}+\frac{\omega+n \Omega_{p}}{u_{00}}\right] a_{3, n}} \\
& =\frac{\Omega^{\prime 2}}{u_{00} \omega_{c}}\left[a_{3 n+1}+a_{3, n-1}-a_{2, n+1}-a_{2, n-1}\right] \\
& +\frac{2 \Omega^{\prime 2}}{\Omega_{p} u_{00}}\left[\frac{\omega_{n+1}}{\beta_{p} u_{00}} a_{3, u+1}+\frac{\omega_{n-1}}{\beta_{p} u_{00}} a_{3, n-1}\right] \\
& {\left[-j \frac{\partial}{\partial z}+\frac{\omega+n \Omega_{p}}{u_{00}}\right] a_{4, n}} \\
& =-\frac{\Omega^{\prime 2}}{u_{00} \omega_{c}}\left[a_{4, n+1}+a_{4, n-1}-a_{1, n+1}-a_{1, n-1}\right] \\
& +\frac{\Omega^{\prime 2}}{\Omega_{p} u_{00}}\left[\frac{\omega_{n+1}}{\beta_{p} u_{00}} a_{4, n+1}+\frac{\omega_{n-1}}{\beta_{p} u_{00}} a_{4, n-1}\right],
\end{aligned}
$$

where $\Omega^{\prime 2} \equiv \eta \beta_{p} E_{p} / 4$ is a pump strength parameter which has the dimension of the square of frequency. The waves which couple together are indicated diagramatically in Fig. 8, and Fig. 9 indicates those waves which can be

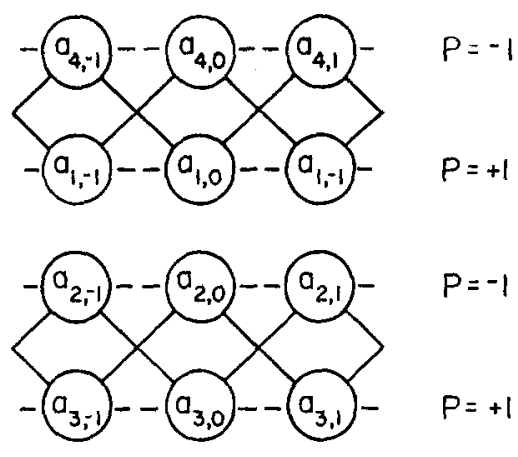

FIG. 8. Diagram showing coupling of modes in an axially symmetric electric pump field. Solid lines indicate active coupling and dashed lines indicate passive coupling.

made to interact strongly through the proper choice of Doppler-shifted pump frequency. The case $\Omega_{p}=0$ is omitted because Eq. (49) breaks down for this situation.

Active coupling of cyclotron modes and a synchronous mode is achieved if the Doppler-shifted pump frequency is equal to the cyclotron frequency. The spatial rate of growth for high-frequency or low-frequency pumping is

$$
\alpha=\Omega^{\prime 2} / u_{00} \omega_{c}=\beta_{p} E_{p} / 4 u_{00} B .
$$

For example, if we consider the case of high-frequency pumping $\left(\omega_{p}>\omega\right)$ and take $\Omega_{p}=+\omega_{c}$, then $a_{1,0}-$ a fast cyclotron wave-is actively coupled to $a_{4,-1}$, a fast synchronous wave, and gain results. The noise can be removed from both modes prior to reaching the pump region by conventional couplers, and a low noise amplifier similar to the Adler quadrupole amplifier is possible. It would, however, probably require two separate couplers to remove the noise from these modes.

A low-frequency or dc-pumped amplifier utilizing active coupling of either a slow cyclotron wave and a fast synchronous wave or a fast cyclotron wave and a slow synchronous wave is also possible according to this analysis. An interesting amplifier would result from the coupling of the $a_{1,0}$ mode to the $a_{4, \pm 1}$ mode with $\omega_{p}=0$. One mode carries positive power and the other negative power, so an energy balance is maintained throughout the interaction region. Rotational energy carried by $a_{1}$ is increased at the expense of the longi-

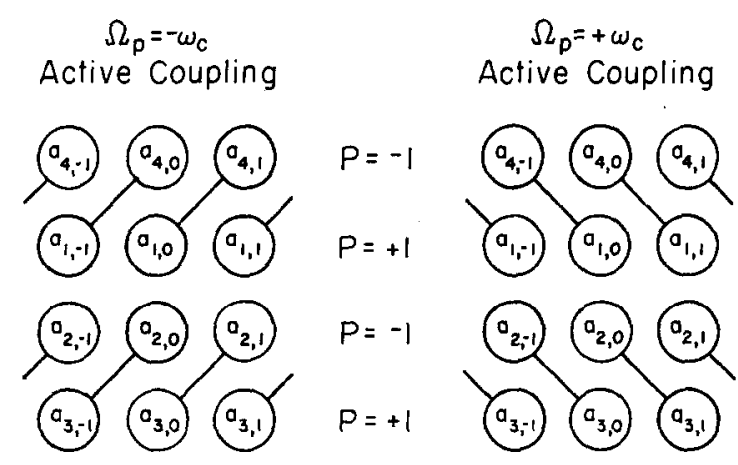

FIG. 9. Diagram showing which modes are strongly interacting in an axially symmetric electric field. 


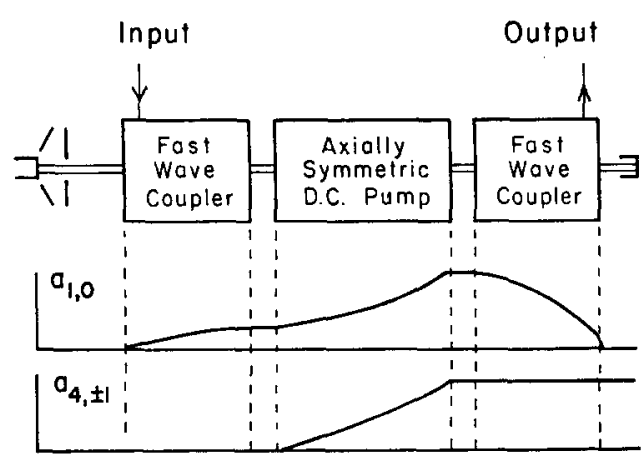

FIG. 10. Schematic of amplifier employing axially symmetric pumping fields. The amplitudes of the relevant modes are plotted below.

tudinal energy carried by $a_{4}$. In a practical amplifier device, an input and output coupler would be used to place on and remove the signal power from the $a_{1}$ mode. The mode amplitudes over the tube length are shown in Fig. 10. After the beam has passed through the output coupler, the only mode present is $a_{4}$. Since this is a mode characterized by uniform axial velocity and no rotational velocity, a depressed collector could be used to great advantage for efficiency improvement.

\section{Periodic Magnetostatic Fields}

Similar coupling relationships are found to exist between the various beam modes when the electrostatic lenses are replaced by magnetostatic lenses. The equations obtained are of the same form as those for the electrostatic case, but the coefficients differ. The mode-coupling diagram of Fig. 8 applies; beam harmonics are generated, and growth can occur between the cyclotron modes and the synchronous mode of opposite power flow.

\section{PUMPING WITH TWO-DIMENSIONAL FIELDS}

The quadrupole field is ideally suited for pumping because the transverse fields vary linearly in $r$. Other types of pumping fields approximate this desired behavior near the axis. One such field is that generated by the longitudinal mode of a transverse-field slow-wave circuit. Consider a circuit of the type shown in Fig. 11. For this case $E_{x}=0$ and $E_{y}=-\left(\Omega^{2} / \eta\right) y \cos \beta_{p} z$ near the axis, where $\Omega^{2}=\eta V_{p} \beta_{p}^{2} / \cosh \beta_{p} a$. On inserting these pumping fields into the mode equations, it is seen that each couples to all four beam modes. Analysis shows that growing waves or beating can be obtained by appropriate coupling to the other beam modes in a fashion analogous to the quadrupole pump case. The rate of gain parameter is somewhat smaller than that for the quadrupole case for a given $V_{p}$. One advantage of this type of pumping is the simpler pump structure. Also, additional flexibility is available in coupling to other modes for noise reduction schemes.

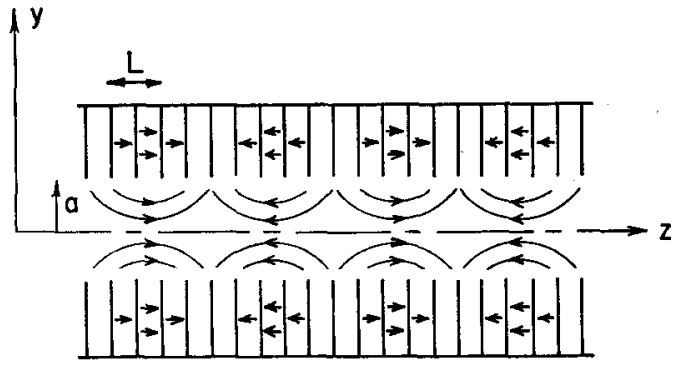

FIG. 11. Sketch of symmetrical finned structure for producing two-dimensional pump fields. The structure and fields are independent of the $x$ coordinate.

The application of this type of pumping field for amplification via dc pumping is possible by coupling the $a_{1}$ mode to either the $a_{2}$ or the $a_{4}$ mode. The mode equations show that active coupling exists between these modes, so for growing waves it is only necessary to provide synchronization.

\section{SUMMARY AND CONCLUSIONS}

A general coupled-mode theory for the transversefield electron beam parametric amplifier has been developed which provides analytic facility and good physical insight into the interaction processes for these tube types. The transverse beam motion in the presence of a pumping field is expressed in terms of an infinite series of modes at the signal frequency and idler frequencies. The interaction process is then described in terms of the coupling of these modes. Generally, the coupling of two particular modes is most important.

This theory has been applied to quadrupole amplifiers, including the low-noise device proposed by Adler and Wade, and the dc-pumped device proposed by Gordon, and others. A clear picture of the interaction processes is obtained, and design equations formulated. A previous paper ${ }^{30}$ has shown that for low-noise amplifiers, the Cuccia coupler is a very efficient noise removal device. The pump theory presented here establishes a sizable source of noise in the pump region, which easily accounts for the observed noise behavior. This effect can be minimized by pumping very weakly. Parametric refrigeration schemes also are investigated and present new possibilities in low-noise devices.

In addition to quadrupole pump fields, axially symmetric periodic fields are proposed and analyzed. Amplifiers of this type have some unique advantages and present a number of new interaction possibilities. The gun region is described by this type of field, and mode coupling in the gun region is found to be very small unless the cyclotron wavelength is greater than the lens focal length.

${ }^{30}$ C. C. Johnson and R. W. Gould, Research Rept. 157, Hughes Research Laboratories, Malibu, California (June, 1960). 